\title{
Embracing model-based designs for dose-finding trials
}

\author{
Sharon B Love ${ }^{*}$, , Sarah Brown ${ }^{2}$, Christopher J Weir ${ }^{3}$, Chris Harbron ${ }^{4}$, Christina Yap ${ }^{5}$,
} Birgit Gaschler-Markefski ${ }^{6}$, James Matcham ${ }^{7}$, Louise Caffrey ${ }^{8}$, Christopher McKevitt $^{9}$, Sally Clive ${ }^{10}$, Charlie Craddock $^{11}$, James Spicer ${ }^{12}$ and Victoria Cornelius ${ }^{13}$

${ }^{1}$ Oxford Clinical Trials Research Unit, Centre for Statistics in Medicine, NDORMS, University of Oxford, Botnar Research Centre, Windmill Road, Oxford OX3 7LD, UK; ${ }^{2}$ Clinical Trials Research Unit, Leeds Institute of Clinical Trials Research, University of Leeds, Leeds LS2 9JT, UK; ${ }^{3}$ Edinburgh Clinical Trials Unit, Usher Institute of Population Health Sciences and Informatics, University of Edinburgh Medical School, Teviot Place, Edinburgh EH8 9AG, UK; ${ }^{4}$ Roche Pharmaceuticals, 6 Falcon Way, Shire Park, Welwyn Garden City AL7 1TW, UK; ${ }^{5}$ Cancer Research UK Clinical Trials Unit, Institute of Cancer and Genomic Sciences, College of Medical and Dental Sciences, University of Birmingham, Birmingham B15 2TT, UK; ${ }^{6}$ Boehringer Ingelheim Pharma GmbH \& Co. KG, Biostatistics and Data Sciences, Birkendorfer Strasse 65, Biberach an der Riss 88400, Germany; ${ }^{7}$ AstraZeneca, DaVinci Building, Melbourn Science Park, Royston SG8 6HB, UK; ${ }^{8}$ School of Social Work and Social Policy, Trinity College Dublin, College Green, Dublin 2, Ireland; ${ }^{9}$ Division of Health and Social Care Research, Faculty of Life Sciences and Medicine, King's College London, London, UK; ${ }^{10}$ Edinburgh Cancer Centre, Western General Hospital, Edinburgh EX4 2XU, UK; ${ }^{11}$ Centre for Clinical Haematology, Haematology - University Hospitals Birmingham NHS Foundation Trust, Queen Elizabeth Hospital, Queen Elizabeth Medical Centre, Birmingham B15 2TH, UK; ${ }^{12}$ Division of Cancer Studies, Bermondsey Wing, Guy's Hospital, Great Maze Pond, London SE1 9RT, UK and ${ }^{13}$ Imperial Clinical Trials Unit, Imperial College London, Stadium House, 68 Wood Lane, London W12 7RH, UK

Background: Dose-finding trials are essential to drug development as they establish recommended doses for later-phase testing. We aim to motivate wider use of model-based designs for dose finding, such as the continual reassessment method (CRM).

Methods: We carried out a literature review of dose-finding designs and conducted a survey to identify perceived barriers to their implementation.

Results: We describe the benefits of model-based designs (flexibility, superior operating characteristics, extended scope), their current uptake, and existing resources. The most prominent barriers to implementation of a model-based design were lack of suitable training, chief investigators' preference for algorithm-based designs (e.g., 3+3), and limited resources for study design before funding. We use a real-world example to illustrate how these barriers can be overcome.

Conclusions: There is overwhelming evidence for the benefits of CRM. Many leading pharmaceutical companies routinely implement model-based designs. Our analysis identified barriers for academic statisticians and clinical academics in mirroring the progress industry has made in trial design. Unified support from funders, regulators, and journal editors could result in more accurate doses for later-phase testing, and increase the efficiency and success of clinical drug development. We give recommendations for increasing the uptake of model-based designs for dose-finding trials in academia.

Dose-finding trials are essential in drug development as they establish a recommended dose for later-phase testing. We need reliable, efficient phase I trial designs for faster, cheaper drug development.
Phase I trials aim to find a recommended dose based on a target/acceptable toxicity level or some other criteria and use algorithm- or model-based designs (Braun, 2014). We focus here

*Correspondence: SB Love; E-mail: sharon.love@csm.ox.ac.uk 
on trials determining the maximum-tolerated dose (MTD), which is the highest dose of drug or treatment that does not cause too many patients unacceptable side effects. Algorithm-based designs, such as the $3+3$ (Carter, 1987), use rules fixed during trial design to select the MTD and allocate patients to a dose level. Dose levels are assigned using information from patients at one dose level. Model-based designs, such as continual reassessment (CRM) (O'Quigley et al, 1990), allocate patients to a dose level using a targeted toxicity rate and a statistical model describing the dosetoxicity relationship between the dose levels. When a new patient is registered to the trial, the model is updated using all available information on all registered patients and the dose for the new patient is agreed using the model-suggested dose as a guideline. Information from every patient at every dose level is used to decide the next dose. The model recommends the final MTD at trial completion.

Although statisticians recommend model-based designs, most phase I trials use algorithm-based designs (Rogatko et al, 2007; van Brummelen et al, 2016). We need to understand why statisticians' endorsement of model-based designs is often ignored (Jaki, 2013; Paoletti et al, 2015) so that we can act appropriately to improve uptake.

We summarise the benefits of model-based designs and statisticians' opinions of why these designs are neglected from the literature. We survey researchers' reasons for avoiding these designs. We demonstrate how to overcome these barriers using a real-world example and provide recommendations and solutions to remove perceived barriers to using model-based designs.

\section{MATERIALS AND METHODS}

Literature review. We conducted a literature review, searching PubMed on 13 May 2015 and Embase on 8 June 2015 for ' $3+3$ ', 'CRM', and general terms. Supplementary Tables A and B show our search strategies.

Survey. We identified four themes in studies examining uptake of adaptive designs and Bayesian methods (Chevret, 2012; Jaki, 2013; Morgan et al, 2014; Dimairo et al, 2015a): resources, knowledge, training, and implementation. We developed survey questions (Supplementary Table C) to identify barriers within these themes. We included one question for statisticians on software and another for other respondents on statistical support.

The survey was sent to clinical academics working with AstraZeneca, chief investigators (CIs) involved in trials reviewed and approved by the Cancer Research UK (CRUK) New Agents Committee (NAC), and International Clinical Trials Methodology Conference delegates (ICTMC 2015) who registered for a dosefinding studies workshop.

The frequency and proportion of each response was calculated for each questionnaire item. The proportion of respondents who considered each item a barrier was calculated by combining the numbers who rated the item 'always' and 'often' or 'strongly agree' and 'agree'.

\section{RESULTS}

Model-based approaches today. Model-based approaches have been neglected since their introduction in the 1990s. They were used in 1.6\% of phase I trials published 1991-2006 (Rogatko et al, 2007), increasing to only $6.4 \%$ by $2012-2014$ (van Brummelen et al, 2016).
Benefits of model-based approaches. Model-based designs for phase I trials offer flexibility, superior operating characteristics, and scope for extension (Le Tourneau et al, 2009).

Flexibility. Model-based approaches allow complete flexibility in defining a target dose-limiting toxicity rate and enable the MTD to be estimated with the required degree of precision. The MTD may therefore be defined as the highest dose with a dose-limiting toxicity rate below the target threshold, with the threshold chosen based on the trial patient population and prior knowledge of the evaluated drug. Model-based designs can accommodate different underlying dose-response curve shapes. Doses can be skipped to accelerate escalation or de-escalation, and new dose levels can be defined during the trial. The risk of dose-limiting toxicity events in later treatment cycles can also be evaluated (Paoletti et al, 2015).

Superior operating characteristics. Across different dose-toxicity curves, model-based designs select the dose with the target doselimiting toxicity rate more often than $3+3$ designs (Thall and Lee, 2003; Boonstra et al, 2015) and expose fewer patients to doses with dose-limiting toxicity rates above or below the target level during the trial (Iasonos et al, 2008; Le Tourneau et al, 2012). The safety of model-based designs is evaluated at the design stage using simulation, with incorporation of overdose control where appropriate, and checking that decisions are sensible. Simulations have shown that more patients are likely to be overdosed or treated at subtherapeutic doses with $3+3$ designs than model-based designs (Babb et al, 1998). Model-based designs also outperform $3+3$ designs when attribution errors for adverse events occur (Iasonos et al, 2012). Unlike $3+3$ designs, model-based designs can accommodate many candidate doses without substantially affecting the designs operating characteristics (Jaki et al, 2013). A CRM design achieved a recommended MTD after a median of three to four fewer patients than a $3+3$ design (Onar et al, 2009).

Extended scope. Model-based approaches can be varied to suit a particular intervention and trial. For example, they can incorporate toxicity grade information (Iasonos et al, 2010; Doussau et al, 2015), combination treatments (Mandrekar et al, 2007), nonbinary end points such as biomarker, pharmacokinetic or pharmacodynamics measures (Calvert and Plummer, 2008), time-to-event information (Cheung and Chappell, 2000), and multiple treatment schedules (O'Quigley and Conaway, 2011).

With so much evidence supporting model-based designs, why do trial teams avoid them?

Possible barriers to model-based approaches. The literature offers many opinions, and little empirical evidence, on why model-based designs are neglected.

Algorithm-based designs such as the $3+3$ design are the most used oncology dose-escalation design and therefore oncologists are exposed to and become familiar with it, and the literature offers many practical examples (Rogatko et al, 2007). Clinicians using $3+3$ designs often informally incorporate available data from lower doses and use their experience of previous trials when deciding dose allocations. Many believe that $3+3$ designs are flexible, practical, functioning phase I designs (Ishizuka and Ohashi, 2001).

Model-based designs are seen as a 'black box' approach to dose escalation that makes clinical interpretation of model parameters difficult during design development. Statistical analysis is needed after each dose cohort, which appears time consuming and complicated. Despite strong counterevidence (O'Quigley, 1999), many believe that model-based designs are less efficient than $3+3$ designs in terms of time-to-complete and numbers treated above 
the MTD (Korn et al, 1994). Our experience is that clinicians also worry that they cannot overrule a model's dose-escalation recommendations, and often cite $3+3$ designs as providing safe, conservative estimates of the MTD.

Clinicians may find model-based designs' need for prior information counterintuitive. As a phase I trial's start is rife with uncertainty, many erroneously believe that the model's required information can only be acquired after the trial starts. The reliability of dose-escalation decision-making is thought to be heavily dependent on weak prior assumptions.

Relative to algorithm-based designs, there are few published practical examples of model-based designs; although the systematic review of Iasonos and O'Quigley (2014) provides references of CRM published trials from 2001 to 2013, Levy et al (2006) and Paoletti et al (2006) are useful descriptions of trials, and Iasonos et al (2015) guides protocol writing.

Setting up model-based designs requires time and expertise. The statistician and CI must interact frequently, requiring access to a statistician and time for design development (Morita et al, 2007; Jaki, 2013; Dimairo et al, 2015b). Even when statistical advice is available, choosing the most appropriate design for a particular trial from the many designs on offer is challenging. Add time constraints due to funding application deadlines, and it is unsurprising that clinicians prefer 'simple', familiar methods (Jaki, 2013; Dimairo et al, 2015b).

Survey results: perceived barriers. We surveyed clinicians, statisticians, researchers, and trial managers to ascertain which of the barriers identified in our literature review are currently affecting the medical research community. We received responses from 14 of the $62(23 \%)$ clinical academics working with AstraZeneca, 22 of the 45 (49\%) CIs involved in trials reviewed and approved by the CRUK NAC, and 43 of the 93 (46\%) participants registered for the ICTMC 2015 workshop giving an overall response rate of $40 \%$ (79 out of 200).

Table 1 summarises the survey participants' disciplines and experience. The majority were CIs (40\%) or statisticians (39\%), representing a range of experience levels. Around half had used non-algorithm-based methods. Of the 30 participating statisticians, $53 \%$ reported access to specialised statistical software to support design and analysis of model-based approaches. Of the 30 participating CIs, $83 \%$ reported access to statistical support to undertake a non-rule-based design.

When designing a new trial, 53\% of the respondents said they always or often considered an alternative to algorithm-based methods. However, $16 \%$ reported a poor experience using alternative designs where reasons given included the reliance of real-time data entry for CRM, which slowed down decision making and less data available on other doses to model the efficacy curve and undertake biomarker exploratory analysis (Supplementary Table D).

Figure 1 shows the proportion of respondents who identified each questionnaire item as a barrier to implementing model-based designs. The top three barriers were lack of training to use alternative approaches to algorithm-based designs (57\%), CIs' preference for $3+3$ designs (53\%), and limited resources for study design before funding (50\%). Many other items were also rated as barriers by a large proportion of the respondents, such as lack of opportunities to apply learnt skills in using alternative approaches to algorithm-based designs and how quickly studies must be designed.

We collected free-text comments to capture other attitudes or barriers (Supplementary Table D). The most common theme was respondents' experience of a model-based study that was slower or larger than a typical $3+3$ design. Other concerns about modelbased designs themes were: difficulties of real-time data capture, limited data on alternative doses for pharmacodynamics, lack of experienced statisticians, and not selecting a safe dose. Improving
Table 1. Characteristics of the survey participants

\begin{tabular}{|c|c|c|c|}
\hline Question & Response & $N$ & $\%$ \\
\hline \multicolumn{4}{|c|}{ Are you $(N=79)$} \\
\hline & $\begin{array}{l}\text { Chief investigator } \\
\text { Funder } \\
\text { Other } \\
\text { Statistician } \\
\text { Trial manager }\end{array}$ & $\begin{array}{r}31 \\
1 \\
14 \\
30 \\
3\end{array}$ & $\begin{array}{r}39 \\
1 \\
18 \\
38 \\
4\end{array}$ \\
\hline
\end{tabular}

How long have you worked with dose-finding studies? (years) $(N=78)$

\begin{tabular}{|l|r|r}
\hline $0-2$ & 10 & 13 \\
$3-5$ & 12 & 15 \\
$6-10$ & 12 & 15 \\
$11-12$ & 14 & 18 \\
$20+$ & 5 & 6 \\
New to topic & 25 & 32
\end{tabular}

Have you ever been involved in a dose-finding study that, rather than using $3+3$ or another rule-based design, used an alternative? $(\mathrm{N}=76)$

\begin{tabular}{|l|l|r|r|}
\hline & No & 37 & 49 \\
& Yes & 37 & 49 \\
& Don't know & 2 & 3 \\
\hline
\end{tabular}

Do you have access to software to support alternative approaches to $\mathbf{3}+\mathbf{3}$ and other rule-based designs? Statisticians only $(N=30)$

\begin{tabular}{|l|r|r}
\hline Don't know & 6 & 20 \\
No & 8 & 27 \\
Yes & 16 & 53
\end{tabular}

Is appropriate statistical support available to you to undertake alternative approaches to $3+3$ and other rule-based designs? Chief investigators only $(N=30)$

\begin{tabular}{|l|r|r} 
Don't know & 2 & 7 \\
No & 3 & 10 \\
Yes & 25 & 83
\end{tabular}

When designing a trial, how often do you consider alternatives to $3+3$ and rule-based designs $(N=77)$

\begin{tabular}{|l|r|r|}
\hline Always & 22 & 29 \\
Often & 19 & 25 \\
Not very often & 15 & 19 \\
Never & 4 & 5 \\
Don't know & 17 & 22 \\
\hline
\end{tabular}

uptake themes were: 'selling' model-based approaches to CIs and funders, accessible software, and consensus on which model-based approach to use.

Resources to support model-based design. Resources exist to help trial designers overcome some of the identified barriers.

Software. UK-based non-industry statisticians have access to free CRM software programmes, such as crmPack, dfcrm, and bcrm (http://cran.r-project.org), $\mathrm{R}$ shiny apps Web Application for simulating operating characteristics of the Bayesian CRM (Wages, 2017), and EWOC (Cedars-Sinai, 2017).

Working groups. The Medical Research Council (MRC) funds a National Network of Hubs for Trial Methodology Research to undertake trials methodology research. The Network's adaptive design working group promotes methodology and supports using innovative designs through workshop-based advice forums, tutorial papers, individualised support, and software development (MRC Hubs for Trials Methodology Research, 2015). The National Institute of Health Research Statistics Group supports translating statistical methods into practice. Its early-phase clinical trial initiative focuses on dose-finding studies and works with academic researchers and industry collaborators (NIHR Statistics Group, 2016). 


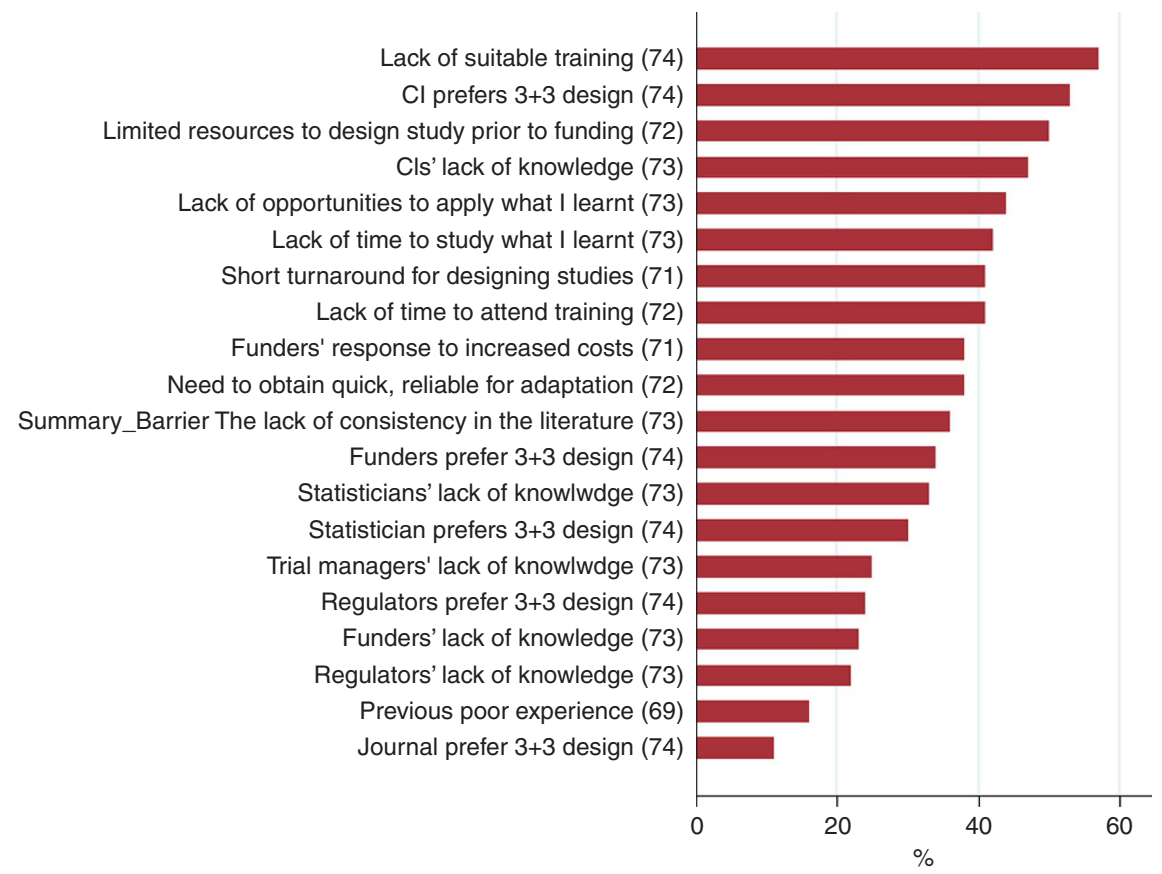

Figure 1. Percentage of respondents identifying each item as a barrier to implementing model-based designs. See Supplementary Table C for all items. $\mathrm{Cl}=$ chief investigator.

\section{Table 2. Pharmaceutical companies' steps to promote model-based designs ${ }^{a}$}

\begin{tabular}{|c|c|c|}
\hline Company & Model-based designs & Key activities \\
\hline AstraZeneca & All early oncology dose-escalation trials since 2014 & $\begin{array}{l}\text { Education programme } \\
\text { Routine trial simulation software } \\
\text { Standard method for prior toxicity-response curves } \\
\text { All possible dose-response scenarios prepared for dose- } \\
\text { escalation meetings }\end{array}$ \\
\hline $\begin{array}{l}\text { Roche Pharmaceutical } \\
\text { Research and Early } \\
\text { Development }\end{array}$ & Standard approach for oncology dose-escalation studies & $\begin{array}{l}\text { Developed R software package, crmPack, for simulating, } \\
\text { visualising, and running CRM studies } \\
\text { Joint scientific forums between statistical and medical } \\
\text { colleagues } \\
\text { Examples of deployed designs }\end{array}$ \\
\hline Boehringer Ingelheim & $\begin{array}{l}\text { Standard approach for dose-finding (two-parameter Bayesian } \\
\text { logistic regression model (Neuenschwander et al, 2008) with } \\
\text { overdose control) }\end{array}$ & $\begin{array}{l}\text { Expert statistics group provides support } \\
\text { Training for statisticians and non-statisticians } \\
\text { Template text for clinical trial protocols } \\
\text { Template R and SAS (SAS Institute Inc., Cary, NC, USA) } \\
\text { programmes for protocols, steering committee meetings, and } \\
\text { clinical trial reports }\end{array}$ \\
\hline
\end{tabular}

Guidelines. As the statistical language used in CRM studies can inhibit understanding, published guidelines indicate which operating characteristics to summarise to help the entire medical, scientific, and statistical team evaluate a proposed design (Iasonos et al, 2015). Other guidelines focus on protocols (Petroni et al, 2017).

Learning from industry. Many pharmaceutical companies have overcome practical barriers to implementing model-based designs, motivated by inaccurate doses from $3+3$ trials causing failed phase II and III studies. Academia can use these companies' experiences (Table 2).

Practical examples. There are few published practical examples of model-based designs (van Meter et al, 2012) or suggested design modifications (Potter, 2002; de Lima et al, 2010; Jones et al, 2012; Wages et al, 2015) to help overcome perceived barriers. In Box 1, we present our experiences developing a CRM-based phase I trial to show an example of the process.

\section{DISCUSSION}

Despite the vast literature outlining the statistical benefits of model-based designs over algorithmic designs, model-based methods are rarely used. In addition, in recent years there have been advances in therapeutic strategies where the relationship between dose and toxicity is less obvious and for which model-based designs are necessary. The literature suggests that time constraints, limited statistical resources, and few published practical examples are the greatest barriers to implementation, and that resources, knowledge, and training are the key to improving uptake. 
Box 1. Planning and executing a phase I trial with a Bayesian design: real-world example

Objective: Perform an open-label single-compound dose-escalation trial to find a new compound's MTD.

The team agreed to consider a model-based approach to explore doses ranging from 0.2 to $120 \mathrm{mg}$. The statisticians explained the proposed design to the clinicians. They emphasised the advantages of a model-based design over a $3+3$ design: flexibility in choosing the next dose and in cohort sizes, and superior operating characteristics. As reliable results were important, the medical trial team agreed to use a Bayesian modelbased approach.

The model-based design needed a prior probability distribution capturing the clinicians' prior beliefs about toxicity at different doses and their uncertainty in these beliefs. The statisticians and clinicians discussed their expectations of the new compound's toxicity. As no clinical data were available, the clinicians estimated the toxicity rates at specific doses for best-case, worst-case, and expected scenarios. As these estimates were based on scant information, they were used to form a minimally informative mixture prior; the information had a small effect on the trial.

The statisticians calculated the prior effective sample size to show the clinicians that the prior's information would not overrule information gathered in the trial. The study design included safety constraints, such as no dose skipping and not recommending doses when the probability of a dose-limiting toxicity was above a threshold.

Hypothetical data scenarios were chosen to reflect potential on-study constellations and the model's recommended escalation decisions were considered. Complete trials were simulated to demonstrate that the model gave reasonable MTD recommendations. As template programmes were used, simulating 3000 trials took just $4-5 \mathrm{~h}$.

To show the team what they could expect in steering committee meetings, Figure 2 and Table 3 were created before the trial started. They represent an example output for a dose-escalation meeting after one hypothetical patient has taken $5 \mathrm{mg}$ without toxicity. The Bayesian model considered doses up to $10 \mathrm{mg}$ safe as they had low probabilities of overtoxicity (Figure 2, top). It classified the dose level most likely to reach the target toxicity range $16-32 \%, 40 \mathrm{mg}$, as too risky for overtoxicity. It recommended testing $10 \mathrm{mg}$ next.

The statisticians explained that the model gives recommendations that the steering committee members can overrule, not binding rules. The statistical report will clearly describe the dose-escalation decisions that were made in the trial.

We surveyed clinicians, statisticians, and trialists interested in dose-finding trials on their opinions of what stops trial teams from using model-based designs. We approached 200 people; $40 \%$ responded. As we targeted a convenient sample of researchers involved or interested in phase I methodology, our results may not be representative of the dose-finding research community. Those acquainted with model-based designs may have been more likely to respond, as $83 \%$ of the clinical respondents reported access to statistical support to implement a model-based approach, and 53\% of the statistician respondents reported access to suitable software. Although the sample may not be a cross-section of the dosefinding community, the opinions of experienced researchers familiar with model-based methods provide valuable insights. Our results agree with previous surveys of similar scope but wider focus (Jaki, 2013; Dimairo et al, 2015b).

We did not identify one obvious barrier. Many barriers were considered important by a large proportion of respondents, including clinician and statistician lack of knowledge; clinician, statistician, and funder preferences; lack of training and time for study design before funding; and funder responses to increased costs. A step change in practice will require a multifold approach targeting funders, clinicians, and statisticians.
Table 3. Toxicity interval probabilities for all prespecified dose levels after one patient has been treated with $5 \mathrm{mg}$

\begin{tabular}{|l|c|c|c|c|}
\hline \multicolumn{5}{|c|}{ Probability of toxicity rate in } \\
\cline { 1 - 2 } $\begin{array}{c}\text { Dose } \\
\text { (mg) }\end{array}$ & $\begin{array}{c}\text { (0-0.16) } \\
\text { Undertoxicity }\end{array}$ & $\begin{array}{c}\text { (0.16-0.33) } \\
\text { Target } \\
\text { toxicity }\end{array}$ & $\begin{array}{c}\text { (0.33-1) } \\
\text { Overtoxicity }\end{array}$ & $\begin{array}{c}\text { Mean } \\
\text { toxicity rate }\end{array}$ \\
\hline 0.2 & 0.889 & 0.065 & $\mathbf{0 . 0 4 6}$ & 0.056 \\
\hline 0.5 & 0.860 & 0.075 & $\mathbf{0 . 0 6 5}$ & 0.071 \\
\hline 1 & 0.828 & 0.089 & $\mathbf{0 . 0 8 3}$ & 0.086 \\
\hline 2.5 & 0.770 & 0.115 & $\mathbf{0 . 1 1 5}$ & 0.112 \\
\hline 5 & 0.717 & 0.128 & $\mathbf{0 . 1 5 5}$ & 0.140 \\
\hline 10 & 0.649 & 0.144 & $\mathbf{0 . 2 0 7}$ & 0.177 \\
\hline 20 & 0.559 & 0.163 & 0.278 & 0.228 \\
\hline 40 & 0.439 & 0.186 & 0.374 & 0.300 \\
\hline 80 & 0.301 & 0.179 & 0.520 & 0.403 \\
\hline 120 & 0.231 & 0.167 & 0.601 & 0.473 \\
\hline Numbers in bold indicate safe, and those in italics indicate unsafe. \\
\hline
\end{tabular}

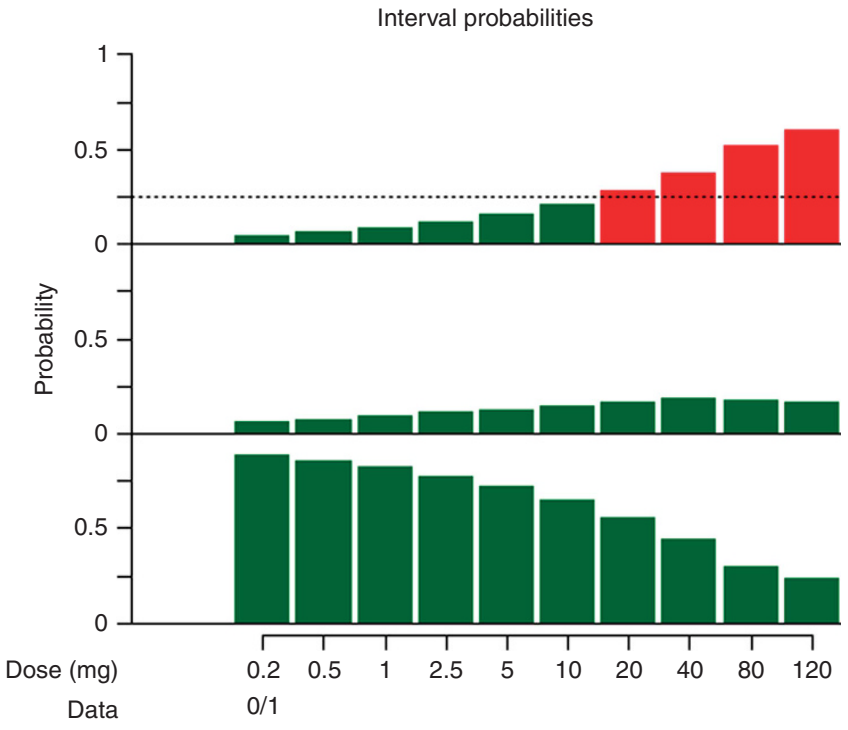

Figure 2. Toxicity interval probabilities for all prespecified dose levels after one patient has been treated with $5 \mathrm{mg}$, showing the probabilities for (top) over-, (middle) target, and (bottom) undertoxicity. With green indicating safe doses and red indicating unsafe doses, this shows the current dose decision can be based solely on overtoxicity since only the overtoxicity graph has red doses. We wish to increase the dose if we can; the current patient took $5 \mathrm{mg}$, but $10 \mathrm{mg}$ would also be safe; thus, the model proposes $10 \mathrm{mg}$ for the next patient.

We discuss the identified barriers to uptake, the progress thus far, and suggestions for facilitating change, referring to our realworld example in Box 1 (see summary in Table 4).

Expectations. Many of our respondents avoided model-based designs as previous attempts had resulted in larger or slower trials than expected. Model-based designs do not necessarily mean smaller phase I trials. Instead, these designs more accurately identify the correct dose for future studies, reducing dose reevaluations and improving efficiency and success in the more expensive later stages of drug development.

Training. Our respondents rated lack of training as the greatest barrier to using model-based designs. The MRC's adaptive design 
Table 4. Summary of recommendations to increase the uptake of model-based designs in academia

\begin{tabular}{|c|c|c|}
\hline & Item & Recommendations \\
\hline Misconceptions & $\begin{array}{l}\text { Cl's disillusioned with the idea that model- } \\
\text { based ideas are more efficient } \\
\text { Perception that regulators prefer } 3+3\end{array}$ & $\begin{array}{l}\text { Address perceptions of 'efficiency' for model-based designs. Communicate that this means } \\
\text { more often accurately identifying the correct dose rather than meaning an individual study will } \\
\text { be shorter in duration or have a lower sample size } \\
\text { Communicate that UK regulators do endorse other trial designs and European regulatory } \\
\text { guidance does not dictate use of a particular trial design }\end{array}$ \\
\hline Training & $\begin{array}{l}\text { Supporting uptake of model-based } \\
\text { designs by statisticians and Cls } \\
\text { Appraisal of studies by funding bodies and } \\
\text { ethics committees } \\
\text { Model-based dose-finding experienced } \\
\text { statisticians contact }\end{array}$ & $\begin{array}{l}\text { While training courses for utilising bespoke expensive software exist, training courses providing } \\
\text { a broad academic introduction to the field and utilising free or inexpensive software need to be } \\
\text { developed } \\
\text { More publications on the practicalities of setting up and running model-based trials } \\
\text { Develop tailored training sessions for key partners to support a thorough scientific appraisal of } \\
\text { proposed designs of phase I trials } \\
\text { Develop a forum for contacting experienced statisticians }\end{array}$ \\
\hline $\begin{array}{l}\text { Design and } \\
\text { evaluation }\end{array}$ & $\begin{array}{l}\text { Lack of time to design and evaluate a } \\
\text { model-based approach }\end{array}$ & $\begin{array}{l}\text { Promote the need for early discussions between } \mathrm{Cl} \text { and statisticians to allow time to develop } \\
\text { and evaluate } \\
\text { Develop software and protocol templates }\end{array}$ \\
\hline
\end{tabular}

working group (MRC Hubs for Trials Methodology Research, 2015) promotes the use of model-based design through publications, workshops, expert advice forums, and individualised support for statisticians. There are some explanatory papers (GarrettMayer, 2006). However, little practical training exists on designing and implementing CRM-based designs throughout a trial's life. More publications on the practicalities are required.

Lack of time. Two frequently reported barriers were how quickly studies are designed and lack of time to study and apply methods. Promoting earlier, frequent discussion of trial ideas between clinicians and statisticians may mitigate these time constraints. Our real-world example shows that ongoing discussion between statisticians and clinicians helps ensure that the final design reflects clinical opinion.

Design evaluation. Our survey highlighted lack of resources for evaluating trial designs as a barrier. The example in Box 1 shows that software templates can speed up design evaluation. Sufficient software training and support during grant development would be very valuable.

Regulators. Over $20 \%$ of our respondents believed that regulators prefer $3+3$ designs and a similar percentage felt regulators lack knowledge of other designs. However, UK regulators do endorse other trial designs, and the European regulatory guidance on firstin-man trials (Committee for Medicinal Products for Human Use, 2007) does not dictate a design (ICH E4, 1994; Hemmings, 2006; European Medicines Agency, 2014; Europeans Medicines Agency, 2015). Experiences from pharmaceutical companies show that model-based designs are readily accepted by health authorities and ethics boards.

A model-based phase I trial design must be described and justified in a clinical trial authorisation application like any other design choice. Regulators evaluate the appropriateness of the chosen method in the application's context. We encourage regulators to make their position clear to clinicians and statisticians.

Funders. Funders drive the academic clinical research agenda by setting strategic health priorities and commissioning research projects. They influence the direction and quality of research, as researchers aim to deliver what funders demand. Funders can play a pivotal role in encouraging better statistical methods in the design and analysis of dose-finding studies by setting strategic objectives, implementing rigorous statistical peer review, and integrating statistical expertise into their processes. We encourage funding bodies and ethics committees to question the use of algorithm-based designs, conduct statistical reviews of all phase I trial applications, and embrace model-based studies.

Ignorance of the benefits of model-based designs and disadvantages of algorithm-based designs is blocking wider implementation of more efficient phase I trial designs. Educating funding bodies, ethics committees, and regulatory agencies via tailored training sessions will enable more scientific appraisal of phase I trial designs. This will provide a greater return on investment: studies will produce more reliable results, increasing the likelihood of successful drug development. We can extend these principles to publications. Journal editors and reviewers should question study designs and how they affect the reliability of dose recommendations for future studies.

\section{CONCLUSION}

By encouraging earlier clinical and statistical discussion, highlighting available training resources and practical examples, and calling for education for funders and other review committees, we hope to help overcome the barriers to model-based designs identified here. Implementing model-based designs will generate more accurate dose recommendations for later-stage testing and increase the efficiency and likelihood of successful drug development.

\section{ACKNOWLEDGEMENTS}

CJW was supported in this work by NHS Lothian via the Edinburgh Clinical Trials Unit. SBL was funded by Grant C5529/ A16895 from the Cancer Research UK. SB was supported in this work by Myeloma UK and Yorkshire Cancer Research. CY was funded by Grant CRUK/12/046 from the Cancer Research UK. JS acknowledges support from the Cancer Research UK and NIHR for the King's Experimental Cancer Medicine Centre. We thank Dr David Wright and Dr Rob Hemmings for information from a regulatory perspective. We also thank Dr Jennifer de Beyer for editing this paper. 


\section{CONFLICT OF INTEREST}

BG-M is an employee of Boehringer Ingelheim Pharma GmbH \& Co. $\mathrm{KG}, \mathrm{CH}$ is an employee of Roche Pharmaceuticals and JM is an employee of AstraZeneca. The other authors declare no other conflict of interest.

\section{REFERENCES}

Babb J, Rogatko A, Zacks S (1998) Cancer phase I clinical trials: efficient dose escalation with overdose control. Stat Med 17(10): 1103-1120.

Boonstra PS, Shen J, Taylor JM, Braun TM, Griffith KA, Daignault S, Kalemkerian GP, Lawrence TS, Schipper MJ (2015) A statistical evaluation of dose expansion cohorts in phase I clinical trials. J Natl Cancer Inst 107(3): pii: dju429.

Braun TM (2014) The current design of oncology phase I clinical trials: progressing from algorithms to statistical models. Chin Clin Oncol 3(1): 2 .

Calvert AH, Plummer R (2008) The development of phase I cancer trial methodologies: the use of pharmacokinetic and pharmacodynamic end points sets the scene for phase 0 cancer clinical trials. Clin Cancer Res 14(12): 3664-3669.

Carter SK (1987) The phase I study. In: Hellmann K, Carter SK (eds). Fundamentals of Cancer Chemotherapy. McGraw-Hill: New York, NY, USA, pp xv, p 527.

Cedars-Sinai (2017) Web-EWOC: Interactive web tool for Designing and Conducting Dose Finding Trials in Cancer, Vol. 2017. Available at: https:// biostatistics.csmc.edu/ewoc/ewocWeb.php (last accessed 26 April 2017).

Cheung YK, Chappell R (2000) Sequential designs for phase I clinical trials with late-onset toxicities. Biometrics 56(4): 1177-1182.

Chevret S (2012) Bayesian adaptive clinical trials: a dream for statisticians only? Stat Med 31(11-12): 1002-1013.

Committee for Medicinal Products for Human Use (2007) Guideline on strategies to identify and mitigate risks for first-in-human clinical trials with investigational medicinal products. European Medicines Agency: London, UK. Doc. ref.emea/chmp/swp/28367/07. Available at: http:// www.ema.europa.eu/docs/en_GB/document_library/Scientific_guideline/ 2009/09/WC500002988.pdf (last accessed 17 January 2017).

de Lima M, Giralt S, Thall PF, Silva LD, Jones RB, Komanduri K, Braun TM, Nguyen HQ, Champlin R, Garcia-Manero G (2010) Maintenance therapy with low-dose azacitidine after allogeneic hematopoietic stem cell transplantation for recurrent acute myelogenous leukemia or myelodysplastic syndrome. Cancer 116(23): 5420-5431.

Dimairo M, Boote J, Julious SA, Nicholl JP, Todd S (2015a) Missing steps in a staircase: a qualitative study of the perspectives of key stakeholders on the use of adaptive designs in confirmatory trials. Trials 16: 430.

Dimairo M, Julious SA, Todd S, Nicholl JP, Boote J (2015b) Cross-sector surveys assessing perceptions of key stakeholders towards barriers, concerns and facilitators to the appropriate use of adaptive designs in confirmatory trials. Trials 16: 585 .

Doussau A, Thiebaut R, Geoerger B, Schoffski P, Floquet A, Le Deley MC, Mathoulin-Pelissier S, Rizzo E, Fumoleau P, Le Tourneau C, Paoletti X (2015) A new approach to integrate toxicity grade and repeated treatment cycles in the analysis and reporting of phase I dose-finding trials. Ann Oncol 26(2): 422-428.

European Medicines Agency (2014) Qualification Opinion of MCP-Mod as an Efficient Statistical Methodology for Model-Based Design and Analysis of Phase II Dose Finding Studies Under Model Uncertainty, Vol. 2014. European Medicines Agency: London, UK. Doc ref EMA/CHMP/SAWP/ 757052/2013. Available at: http://www.ema.europa.eu/docs/en_GB/ document_library/Regulatory_and_procedural_guideline/2014/02/ WC500161027.pdf (last accessed 17 January 2017).

Europeans Medicines Agency (2015) Report from Dose Finding Workshop, Vol. 2015. European Medicines Agency: London, UK. Doc ref EMA/ 117491/2015. Available at: http://www.ema.europa.eu/docs/en_GB/ document_library/Report/2015/04/WC500185864.pdf (last accessed 16 January 2017).

Garrett-Mayer E (2006) The continual reassessment method for dose-finding studies: a tutorial. Clin Trials 3(1): 57-71.

Hemmings R (2006) Philosophy and methodology of dose-finding - a regulatory perspective. In: Chevret S (ed). Statistical Methods for DoseFinding Experiments. Wiley: Chichester, UK.
Iasonos A, Gonen M, Bosl GJ (2015) Scientific review of phase I protocols with novel dose-escalation designs: how much information is needed? J Clin Oncol 33(19): 2221-2225.

Iasonos A, Gounder M, Spriggs DR, Gerecitano JF, Hyman DM, Zohar S, O'Quigley J (2012) The impact of non-drug-related toxicities on the estimation of the maximum tolerated dose in phase I trials. Clin Cancer Res 18(19): 5179-5187.

Iasonos A, O'Quigley J (2014) Adaptive dose-finding studies: a review of model-guided phase I clinical trials. J Clin Oncol 32(23): 2505-2511.

Iasonos A, Wilton AS, Riedel ER, Seshan VE, Spriggs DR (2008) A comprehensive comparison of the continual reassessment method to the standard $3+3$ dose escalation scheme in phase I dose-finding studies. Clin Trials 5(5): 465-477.

Iasonos A, Zohar S, O'Quigley J (2010) Two-stage continual reassessment method that determines dose escalation based on individual toxicity grades. Clin Trials 7: 496.

ICH E4 (1994) Dose-Response Information to Support Drug Registration, Vol. 1994. The International Council for Harmonisation of Technical Requirements for Pharmaceuticals for Human Use (ICH): Geneva, Switzerland. Available at: http://www.ich.org/fileadmin/Public_Web_Site/ ICH_Products/Guidelines/Efficacy/E4/Step4/E4_Guideline.pdf (last accessed 16 January 2017).

Ishizuka N, Ohashi Y (2001) The continual reassessment method and its applications: a Bayesian methodology for phase I cancer clinical trials. Stat Med 20(17-18): 2661-2681.

Jaki T (2013) Uptake of novel statistical methods for early-phase clinical studies in the UK public sector. Clin Trials 10(2): 344-346.

Jaki T, Clive S, Weir CJ (2013) Principles of dose finding studies in cancer: a comparison of trial designs. Cancer Chemother Pharmacol 71(5): 1107-1114.

Jones DR, Moskaluk CA, Gillenwater HH, Petroni GR, Burks SG, Philips J, Rehm PK, Olazagasti J, Kozower BD, Bao YD (2012) Phase I trial of induction histone deacetylase and proteasome inhibition followed by surgery in non-small-cell lung cancer. J Thorac Oncol 7(11): 1683-1690.

Korn EL, Midthune D, Chen TT, Rubinstein LV, Christian MC, Simon RM (1994) A comparison of 2 phase-I trial designs. Stat Med 13(18): 1799-1806.

Le Tourneau C, Gan HK, Razak AR, Paoletti X (2012) Efficiency of new dose escalation designs in dose-finding phase I trials of molecularly targeted agents. PLoS ONE 7(12): e51039.

Le Tourneau C, Lee JJ, Siu LL (2009) Dose escalation methods in phase I cancer clinical trials. J Natl Cancer Inst 101(10): 708-720.

Levy V, Zohar S, Bardin C, Vekhoff A, Chaoui D, Rio B, Legrand O, Sentenac S, Rousselot P, Raffoux E, Chast F, Chevret S, Marie JP (2006) A phase I dose-finding and pharmacokinetic study of subcutaneous semisynthetic homoharringtonine (ssHHT) in patients with advanced acute myeloid leukaemia. Br J Cancer 95(3): 253-259.

Mandrekar SJ, Cui Y, Sargent DJ (2007) An adaptive phase I design for identifying a biologically optimal dose for dual agent drug combinations. Stat Med 26(11): 2317-2330.

Morgan CC, Huyck S, Jenkins M, Chen L, Bedding A, Coffey CS, Gaydos B, Wathen JK (2014) Adaptive design: results of 2012 survey on perception and use. Ther Innov Regul Sci 48(4): 473-481.

Morita S, Toi M, Saji S, Iwata H, Ohno S, Ito Y, Kobayashi T, Hozumi Y, Sakamoto J (2007) Practical application of the continual reassessment method to a phase I dose-finding trial in advanced breast cancer. Drug Inf J 41(6): 691-700.

MRC Hubs for Trials Methodology Research (2015) Network Hubs: Adaptive Designs, Vol. 2016. Medical Research Council: London, UK. Available at: http://www.methodologyhubs.mrc.ac.uk/research/working-groups/ adaptive-designs/ (last accessed 8 December 2016).

Neuenschwander B, Branson M, Gsponer T (2008) Critical aspects of the Bayesian approach to phase I cancer trials. Stat Med 27(13): 2420-2439.

NIHR Statistics Group (2016) Research: Early Phase Trials, Vol. 2016. King's College London: London, UK. Available at: http://statisticsgroup.nihr.ac.uk/research/early-phase-clinical-trials (last accessed 8 December 2016).

O'Quigley J (1999) Another look at two phase I clinical trial designs. Stat Med 18(20): 2683-2690.

O'Quigley J, Conaway M (2011) Extended model-based designs for more complex dose-finding studies. Stat Med 30(17): 2062-2069.

O'Quigley J, Pepe M, Fisher L (1990) Continual reassessment method: a practical design for phase 1 clinical trials in cancer. Biometrics 46(1): $33-48$. 
Onar A, Kocak M, Boyett JM (2009) Continual reassessment method vs. traditional empirically based design: modifications motivated by phase I trials in pediatric oncology by the Pediatric Brain Tumor Consortium. J Biopharm Stat 19(3): 437-455.

Paoletti X, Baron B, Schoffski P, Fumoleau P, Lacombe D, Marreaud S, Sylvester R (2006) Using the continual reassessment method: lessons learned from an EORTC phase I dose finding study. Eur J Cancer 42(10): $1362-1368$.

Paoletti X, Ezzalfani M, Le Tourneau C (2015) Statistical controversies in clinical research: requiem for the $3+3$ design for phase I trials. Ann Oncol 26(9): 1808-1812.

Petroni GR, Wages NA, Paux G, Dubois F (2017) Implementation of adaptive methods in early-phase clinical trials. Stat Med 36(2): 215-224.

Potter DM (2002) Adaptive dose finding for phase I clinical trials of drugs used for chemotherapy of cancer. Stat Med 21(13): 1805-1823.

Rogatko A, Schoeneck D, Jonas W, Tighiouart M, Khuri FR, Porter A (2007) Translation of innovative designs into phase I trials. J Clin Oncol 25(31): 4982-4986.

Thall PF, Lee SJ (2003) Practical model-based dose-finding in phase I clinical trials: methods based on toxicity. Int J Gynecol Cancer 13(3): 251-261. van Brummelen EMJ, Huitema ADR, van Werkhoven E, Beijnen JH, Schellens JHM (2016) The performance of model-based versus rulebased phase I clinical trials in oncology. J Pharmacokinet Pharmacodyn 43: 235-242.

van Meter E, Slone S, Weiss H, Reynolds J, Hayslip J (2012) Implementation of likelihood-based continual reassessment method designs in dose finding trials. Clin Trials 9: 550.

Wages NA (2017) Web Application for Simulating Operating Characteristics of the Bayesian CRM: Bayesian Continual Reassessment Method for Phase I Clinical Trials. Available at: https://uvatrapps.shinyapps.io/crmb/ (last accessed 27 April 2017).

Wages NA, Read PW, Petroni GR (2015) A phase I/II adaptive design for heterogeneous groups with application to a stereotactic body radiation therapy trial. Pharm Stat 14(4): 302-310.

(c) (i) This work is licensed under the Creative Commons Attribution 4.0 International License. To view a copy

(C) The Author(s) named above 2017

Supplementary Information accompanies this paper on British Journal of Cancer website (http://www.nature.com/bjc) 